\title{
A model for tear film dynamics during a realistic blink
}

Kara L. Maki ${ }^{1}$, William D. Henshaw ${ }^{2}$, Alex McManus ${ }^{1}$, Richard J. Braun ${ }^{3}$, Dylan M. Chapp $^{3}$, Tobin A. Driscoll ${ }^{3}$

${ }^{1}$ School of Mathematical Sciences, Rochester Institute of Technology, Rochester, NY, USA, ${ }^{2}$ Department of Mathematical Sciences, Rensselaer Polytechnic Institute, Troy, NY, USA, ${ }^{3}$ Department of Mathematical Sciences, University of Delaware, Newark, DE, USA

Keywords: blink, tear breakup, tear film, thin film equation

\section{Background and purpose}

Each time one blinks, a stable tear film (TF) must reestablish itself on the ocular surface to ensure the function and health of the eye. Although the formation of the TF has been extensively studied both experimentally and theoretically, ${ }^{1,2}$ the influence of the lid dynamics on the TF formation is still not fully understood. Experimental instrumentation does not yet have the capability to estimate the TF thickness in vivo over the entire front of the eye, especially near the lids during a blink, where the eyelashes obstruct the view of the ocular surface. Additionally, a realistic blinking eyeshaped domain presents challenges in approximating the motion of the TF in theoretical studies. In this work, we overcome these theoretical challenges by implementing a moving overset grid method to study the influence of the lid motion on the formation of TF.

\section{Methods}

The realistic eye-shaped domain is described by curves fit to the lid margin of a partial blinking eye. Figure 1 shows one frame from a video of a partial blink, and the curve fit to the lid margin. In each frame of the video, a Fourier series in space, with fifteen nodes, is fit using a least-squares approximation to the lid margin data. Next, each coefficient of the Fourier series describing the lid margin in each frame of the video is treated as a time series of data and fit to a Fourier series in time with five nodes. Thus, the shape of the eye, and boundary of our domain, $\partial \Omega(t)$, can be characterized at any given time during the partial blink.

Correspondence: School of Mathematical Sciences, Rochester Institute of Technology, Rochester, NY, USA.

E-mail: kmaki@rit.edu 


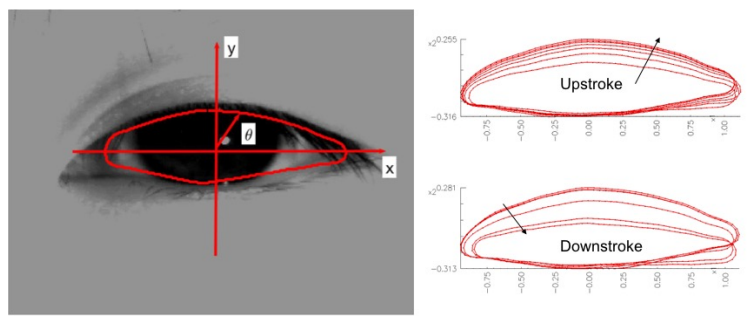

Fig. 1. (Left) Frame from a video of a blink with the curve approximating the lid margin. (Right) A family of curves approximating the lid margin during the upstroke (top) and the downstroke (bottom).

Here, we describe our mathematical model for the evolution of the aqueous layer thickness of the TF, denoted by $z=h(x, y, t)$. We assume the TF is aqueous tears with fluid properties matching water and viscosity $\mu$. In this work, we ignore gravitational effects. On the ocular surface, approximated as the plane, $z=0$, we assume no slip and impermeability. At the TF/air interface, $z=h(x, y, t)$, we assume the lipid layer has rendered the surface tangentially immobile, and the difference in the normal stresses across the interface is equal to capillary pressure due to surface tension $\sigma$. The characteristic length scale for the ocular surface is $L=2 \mathrm{~cm}$, whereas the characteristic thickness of the tears is $H=16 \mu \mathrm{m} .^{3}$ The aspect ratio $\epsilon=H / L=8 \times 10^{-4}$ arises as a small parameter when we nondimensionalize the Navier-Stokes equations; we then apply lubrication theory ${ }^{4}$ to derive a thin film equation for the TF thickness:

$$
\begin{aligned}
\frac{\partial h}{\partial t} & =\nabla \cdot\left(\frac{h^{3}}{12} \nabla p\right), \\
p & =-S \Delta h,
\end{aligned}
$$

on $(x, y) \in \Omega(t)$, where $p(x, y, t)$ denotes the pressure of the aqueous tears. The nondimensional parameter $S=\epsilon^{3} \sigma / \mu V \approx 7.8 \times 10^{-7}$ characterizes the relative importance of viscous and capillary effects. Changes in $h$ are determined by spatial variations in the aqueous volumetric flux, $\mathbf{Q}=-\frac{h^{3}}{12} \nabla p$. Note that we solve the problem as a system of nonlinear partial differential equations (PDEs) to facilitate the numerical approximation of the solution.,

To close the system of PDEs, we enforce boundary conditions (BCs) to mimic realistic TF dynamics. The first specifies the TF meniscus width at the lid margins; specifically, we set $\left.h\right|_{\partial \Omega(t)}=h_{0}=13$; dimensionally, $208 \mu \mathrm{m}$ falls into the range of reported average values for the tear meniscus width $h_{0}$ from $6 \times 10^{-5} \mathrm{~m}^{7,8}$ to $3.65 \times 10^{-4}$ $\mathrm{m} .{ }^{9}$ The second $\mathrm{BC}$ specifies the flux of fluid entering and exiting the exposed ocular surface; the flux may be chosen to mimic the lacrimal system as characterized by Doane. ${ }^{10}$ Specifically, we may specify the aqueous volumetric flux, $\mathrm{Q}$, normal to the boundary:

$$
\mathbf{Q}(\theta, t) \cdot \mathbf{n}_{\partial \Omega}(\theta, t)=Q_{l m}(\theta, t)+Q_{l g}(\theta, t)+Q_{p}(\theta, t),
$$


where $\mathbf{n}_{\partial \Omega}(\theta, t)$ is the normal vector to lid margins at position $\theta$ and time $t ; \theta$ is the angle relative to the positive $x$-axis, with the $y$-axis at $\theta=\pi ; Q_{l m}(\theta, t)$ is the flux proportional to lid motion (FPLM); $Q_{l g}(\theta, t)$ is the lacrimal gland influx; and $Q_{p}(\theta, t)$ is the punctal drainage efflux. In this work, we assume that during lid motion, the lacrimal gland supply and punctal drainage are not active, i.e., $Q_{l g}=Q_{p}=0$, and the FPLM is given by:

$$
Q_{l m}(\theta, t)=h_{l m} \mathbf{v}_{\partial \Omega}(\theta, t) \cdot \mathbf{n}_{\partial \Omega}(\theta, t),
$$

where $\mathbf{v}_{\partial \Omega}(\theta, t)$ is the velocity of the lid, found by taking the time derivative of the double Fourier series representation of the lid margin. ${ }^{11}$ When $h_{l m}=h_{0}$, the tear volume is conserved during the blink. When $h_{l m}=h_{0}-h_{e} / 2$, we assume there exists a layer of aqueous tears of thickness $h_{e}$ under the lid margins and there is a Couette flow between the unexposed eye surface and the lids that enters and exits the TF during the blink. ${ }^{11}$ In the results presented below, $h_{e}=1.6(12 \%$ of the tear meniscus width).

The initial TF thickness and pressure distributions are shown in Figure 2. The initial TF distribution is found by solving $p=-S \Delta h$, where the pressure distribution is specified by $p=-S\left(3.5 x^{4}+2 y^{2}+1\right)$, chosen to mimic features seen in vivo. The initial dimensional tear volume is $21.25 \mu \mathrm{l}$ which is eight times the $2.45 \mu \mathrm{l}$ volume of the TF estimated in vivo on an open eye. ${ }^{12}$
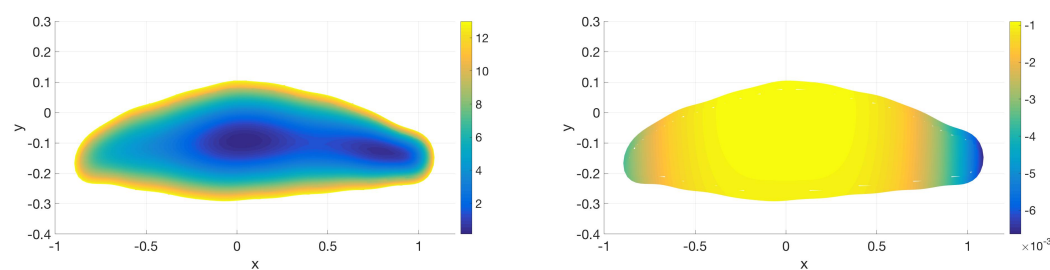

Fig. 2. The initial TF thickness (left) and pressure distribution (right).

The evolution equations (1) and (2) are approximated using an overset moving grid method in Overture (http://www.overtureframework.org). The eye-shaped domain is discretized using moving overlapping grids (a collection of logically rectangular curvilinear component grids) where a boundary-fitted grid is used to represent the moving lids, and this grid overlaps with a stationary background Cartesian grid. ${ }^{13,14}$ Each component grid is defined by a smooth mapping from computational space (the unit square) to the physical domain. The governing equations are transformed to the unit square and then discretized in space using standard finite difference approximations. On the moving boundary-fitted grid, the equations are solved in a moving coordinate system. The solutions on the different component grids are connected by interpolation. The nonlinear equations are advanced in time using a second-order accurate backward-differentiation (BDF) scheme. The numerical accuracy has been verified on test problems with exact solutions. ${ }^{15}$ 


\section{Results}

We validated the performance of the numerical approach on the current problem by analyzing the conservation of the TF volume during the blink with no flux BCs. The volume change is less than $2 \%$ of the initial tear volume for the entire blink cycle.

We now examine how different flux BCs, linked to lid motion and lid speed, affect the tear formation and subsequent TF breakup times.

\subsection{TF formation: the upstroke}

We first consider the no flux $\mathrm{BC}$ where TF volume is conserved. The left panel in Figure 3 shows the evolution of the TF during the upstroke. As the upper lid opens, the TF thins along the lid margin as more eye surface is exposed, thus creating the black line (shown as dark blue). The local thinning near the upper lid is consistent with prior work with 1-D models. ${ }^{2}$ However, now we can see how spatial variation in the lid motion affects TF formation. In this model, the black line first forms in the center of the eye $(\theta \approx \pi / 2)$ and then extends towards the temporal canthus $(\theta \approx 0)$. The thinning continues as more eye surface is exposed, causing the radius of curvature of the upper meniscus to decrease. This creates an even lower pressure region in the upper meniscus and accelerates meniscus-induced thinning.

The right panel in Figure 3 shows the evolution of the TF during the upstroke, when aqueous tears are supplied from under the lids via the FPLM BC. The supplied tears suppress the formation of the black line along the upper lid margin. We still see evidence of the meniscus-induced thinning in the corners, with the black line first appearing in the temporal canthus.

The different flux BCs result in different TF distributions at the end of the upstroke and the end of the downstroke. Figure 4 compares the TF thickness after the upstroke $(t=1)$ and a complete blink ( $t=3 / 2$ ). Unsurprisingly, in the upper meniscus, the no flux BC simulation results in a thinner TF than with FPLM BC. In the center of the exposed area, the difference in the thickness at the end of upstroke is nearly zero; the effect of the flux BCs is localized to the menisci regions. Interestingly, at the end of the downstroke, in the center of the exposed area, the no flux BC yields a thicker TF than for the FPLM BC. The "snow plow" effect of the upper lid during the downstroke causes more global thickness variation.

\subsection{Tear relaxation: the interblink}

Figure 5 shows the continuation of the tears supplied under lids during the upstroke into the interblink period. After completion of the upstroke, the eye remains open for $\sim 2$ seconds. The figure shows the TF thickness and the aqueous flux after the eye remains open for approximately 1.5 seconds. Meniscus-induced thinning continues to form the black line. As found in the prior work on a stationary eye-shaped domain, 4,16 the tears in the lower meniscus collect near the canthi due to the increased curvature of the lid margin. An interesting flow pattern develops in the upper meniscus. In the 

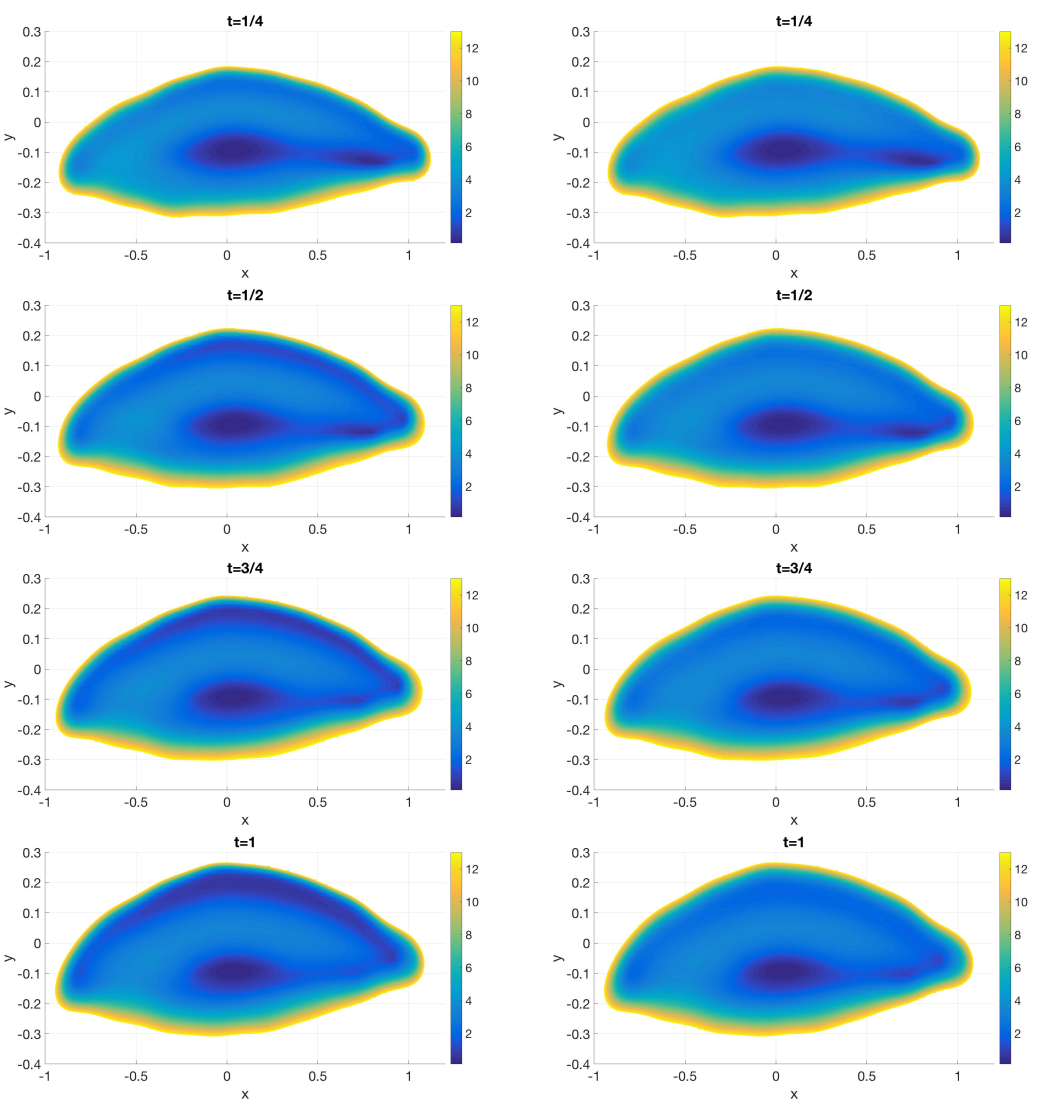

Fig. 3. (Left) TF thickness during the upstroke, when the volume is conserved (no flux BC). (Right) TF thickness using the FPLM BC, where the upper lid exposes aqueous tears from under the lid.

nasal canthus, the tears continue to flow into the upper meniscus region towards the temporal canthus. Unlike previous work on the stationary domain, we find the lowerpressure region that forms in the upper meniscus during the upstroke continues to draw fluid into the upper meniscus after the lid motion has stopped. These spatial variations in the upper tear meniscus curvature at the end of the upstroke (and consequently, the pressure) depend on spatial variations in the lid speed and influxes. We note that adding punctal drainage and lacrimal gland supply may reverse that flow.

\section{Conclusions and future perspectives}

We find the formation of the TF during the upstroke is sensitive to the lid motion and the tear supply from under lids. The local tear film thinning in the upper meniscus is dependent on the rate of the exposure of the eye surface and the influxes/effluxes. 

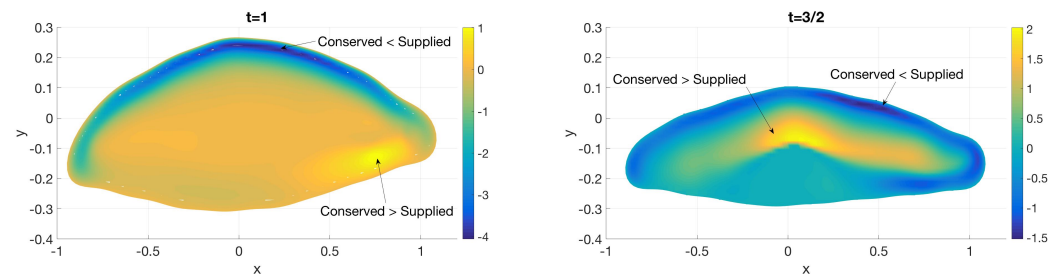

Fig. 4. The difference in the thickness distribution between the no flux and FLPM BCs. (Left) The end of the upstroke $(t=1)$. (Right) The end of the downstroke $(t=3 / 2)$.
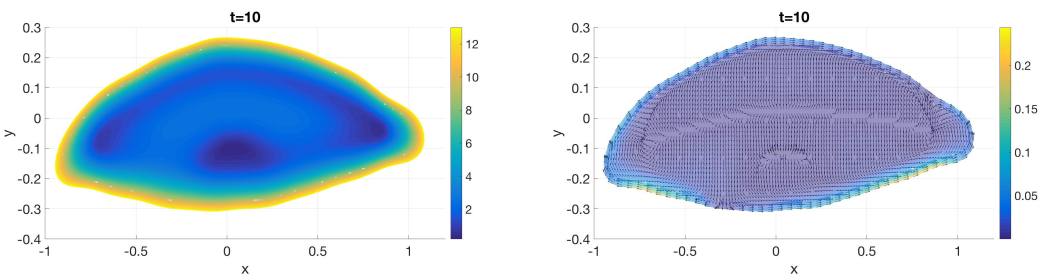

Fig. 5. Relaxation of the TF during the interblink. The TF thickness and aqueous flux after the eye has been open for 1.5 seconds.

Any spatial variation in the local tear film thinning rates establishes a flow within upper meniscus region. During the interblink period, we found that the curvature of the lid margin can accelerate the formation of the black lines by establishing low-pressure regions in the menisci. In future work, the model described here should be extended to include gravitational and evaporative effects, and more realistic influxes/effluxes to provide insight into the tear film dynamics near the upper lid during a blink, a region that is difficult to image.

\section{Acknowledgements}

This material is based upon work supported by the National Science Foundation (NSF) under Grant Numbers DMS 1412141 (KLM) and 1412085 (RJB and TAD), and does not necessarily represent the official view of the NSF.

\section{References}

1. Braun R, King-Smith P, Begley C, Li L, Gewecke N. Dynamics and function of the tear film in relation to the blink cycle. Prog Ret Eye Res. 2015;45: 132 -164.

2. Braun RJ. Dynamics of the tear film. Annu Rev Fluid Mech. 2012;44: 267-297.

3. King-Smith E, Fink B, Hill R, Koelling K, Tiffany J. The thickness of the tear film. Curr Eye Res. 2004;29(4-5): 357-368.

4. Maki KL, Braun RJ, Ucciferro P, Henshaw WD, King-Smith PE. Tear film dynamics on an eye-shaped domain. Part 2. Flux boundary conditions. J Fluid Mech. 2010;647: 361-390. 
5. Heryudono A, Braun RJ, Driscoll TA, Cook LP, Maki KL, King-Smith PE. Single-equation models for the tear film in a blink cycle: Realistic lid motion. Math Med Biol. 2007;24: 347-377.

6. Maki KL, Braun RJ, Driscoll TA, King-Smith PE. An overset grid method for the study of reflex tearing. Math Med Biol. 2008;25: 187-214.

7. Mainstone JC, Bruce AS, Golding TR. Tear meniscus measurement in the diagnosis of dry eye. Curr Eye Res. 1996;15(6): 653-661.

8. Golding TR, Bruce AS, Mainstone JC. Relationship between tear-meniscus parameters and tear-film breakup. Cornea. 1997;16(6): 649-661.

9. Gaffney E, Tiffany J, Yokoi N, Bron A. A mass and solute balance model for tear volume and osmolarity in the normal and the dry eye. Prog Ret Eye Res. 2010;29(1): 59 -78.

10. Doane MG. Blinking and the mechanics of the lacrimal drainage system. Ophthalmology. 1981;88: 844851.

11. Jones MB, Please CP, McElwain DLS, Fulford GR, Roberts AP, Collins MJ. Dynamics of tear film deposition and drainage. Math Med Bio. 2005;22: 265-288.

12. Mishima S, Gasset A, Klyce SD, Baum JL. Determination of tear volume and tear flow. Ophthalmol Vis Sci. 1966;5: 264-276.

13. Chesshire G, Henshaw WD. Composite overlapping meshes for the solution of partial differential equations. J Comput Phys. 1990;90: 1-64.

14. Henshaw WD, Schwendeman DW. Moving overlapping grids with adaptive mesh refinement for highspeed reactive and non-reactive flow. J Comput Phys. 2006;216(2): 744 -779.

15. Roache PJ. Verification of codes and calculations. AIAA J. 1998;36(5): 696-702.

16. Li L, Braun RJ, Maki KL, Henshaw WD, King-Smith PE. Tear film dynamics with evaporation, wetting and time-dependent flux boundary condition on an eye-shaped domain. Phys Fluids. 2014;26: 052101. 